\title{
Self-assembly of corrole trimers in solution and at the solid-liquid interface $\dagger$
}

\author{
Richard van Hameren, ${ }^{a}$ Johannes A. A. W. Elemans, ${ }^{* a}$ Dagmara Wyrostek, ${ }^{b}$ Mariusz Tasior, ${ }^{b}$ \\ Daniel T. Gryko, ${ }^{* b}$ Alan E. Rowan ${ }^{a}$ and Roeland J. M. Nolte ${ }^{a}$
}

\author{
Received 22nd July 2008, Accepted 7th October 2008 \\ First published as an Advance Article on the web 17th October 2008 \\ DOI: $10.1039 / \mathrm{b812518j}$
}

\begin{abstract}
The self-assembly of corrole trimers in solution and at solid-liquid interfaces is a process that depends on dewetting, hydrogen bonding and $\pi-\pi$ interactions between the molecules forming columnar stacks, and lateral interactions between these stacks to generate higher order assemblies.
\end{abstract}

The bottom-up approach of self-assembling molecules at a surface is of fundamental importance for constructing new materials and devices. ${ }^{1,2}$ By combining the principles of supramolecular chemistry with physical dewetting, surfaces can be patterned with well-defined arrays of molecules in dimensions ranging from nanometers to even millimeters. ${ }^{3}$ Currently, a major challenge is the implementation of function into these patterns, such as switching, ${ }^{4}$ electronic ${ }^{5}$ and photovoltaic behaviour, ${ }^{6}$ or catalysis. ${ }^{7,8}$ In particular with respect to those properties, the construction of well-defined arrays of chromophoric molecules, such as porphyrins, phthalocyanines and other porphyrinoids, is appealing. ${ }^{9}$ Recently, we developed a method to construct periodically patterned surfaces of linear assemblies simply by drop-casting a chloroform solution of a disk-shaped porphyrin trimer, having porphyrins connected to a central benzene core via amide linkers and functionalised with meso- $n$-dodecoxyphenyl groups, onto a mica surface and allowing the solvent to evaporate. ${ }^{10}$ The patterns, which cover areas extending to several square millimetres, consist of equidistant single molecule wide columnar stacks of the trimer, and are formed by a delicate balance between physical dewetting processes and highly directional supramolecular selfassembly. The latter process is governed by a combination of intermolecular hydrogen bonding between the amide moieties and $\pi-\pi$ stacking interactions between the porphyrins. Upon drop-casting related porphyrin trimers with chiral alkyl chains, linear patterns of chiral stacks were formed. ${ }^{11}$ When instead of chloroform $n$-hexane was used as the solvent, self-assembly occurred in solution, and upon drop-casting, single molecule thick fibres were deposited randomly onto the surface. Intrigued by the well-defined self-assembly behaviour of these porphyrin trimers, we decided to investigate the behaviour of other chromophores when they are subject to selfassembly and dewetting, with the aim to gain better insight into structure-property relationships. Our attention was attracted by the corroles, since self-assembly of these chromophores is unprecedented

${ }^{a}$ Institute for Molecules and Materials, Radboud University Nijmegen, Toernooiveld 1, 6525 ED Nijmegen, The Netherlands. E-mail: J.Elemans@science.ru.nl

${ }^{b}$ Institute of Organic Chemistry of the Polish Academy of Sciences, Kasprzaka 44/52, 01-224 Warsaw, Poland.E-mail: daniel@icho.edu.pl $\dagger$ Electronic supplementary information (ESI) available: Synthesis and analytical data of the compounds and experimental procedures. See DOI: $10.1039 / \mathrm{b} 812518 \mathrm{j}$ and because they display a particularly unique combination of chemical and physical properties (i.e., high fluorescence quantum yield, ability to generate singlet oxygen, singlet and triplet excited states with well-characterised spectra, intriguing coordination chemistry, low oxidation and reduction potentials). ${ }^{12-15}$ In contrast to many other members of the porphyrinoid family, they have one additional advantage: the methodology of their synthesis has reached a mature state in recent years. ${ }^{16}$ Here we present the synthesis of corrole trimer 1 (Chart 1), and its self-assembly behaviour in solution and at solid-liquid interfaces.

The initial strategy towards $\mathbf{1}$ was based on 4-dodecyloxybenzaldehyde as a building block. Although the corresponding dipyrromethane could be synthesised, its further transformations led to unstable corroles in very small yields (see ESI $\dagger$ ). Consequently, we decided to replace the building block with its perfluorinated analogue 3 (Scheme 1). Because of their electron-withdrawing character, the introduction of eight fluorine atoms into the final corrole molecule should increase its stability. ${ }^{15}$ In addition, the same effect enhances the stability of the corresponding dipyrromethane, which should lead to increased yields of the corrole. ${ }^{16,17}$ Commercially available 2,3,5,6tetrafluorophenol (2) was alkylated and formylated using standard procedures. Aldehyde $\mathbf{3}$ was then transformed into the corresponding dipyrromethane 4 in 96\% yield, using Lindsey's procedure. ${ }^{18}$ The trans- $\mathrm{A}_{2} \mathrm{~B}$-corrole synthesis from dipyrromethane $\mathbf{4}$ and 4-phthalimidobenzaldehyde (5) ${ }^{19}$ under TFA $/ \mathrm{CH}_{2} \mathrm{Cl}_{2}$ conditions ${ }^{17}$ led to the corresponding corrole in $5 \%$ yield. The final deprotection with hydrazine hydrate afforded 6 almost quantitatively. Corrole trimer $\mathbf{1}$ was then synthesised from $\mathbf{6}$ and trimesoyl chloride in dichloromethane, using triethylamine as a base. After purification by

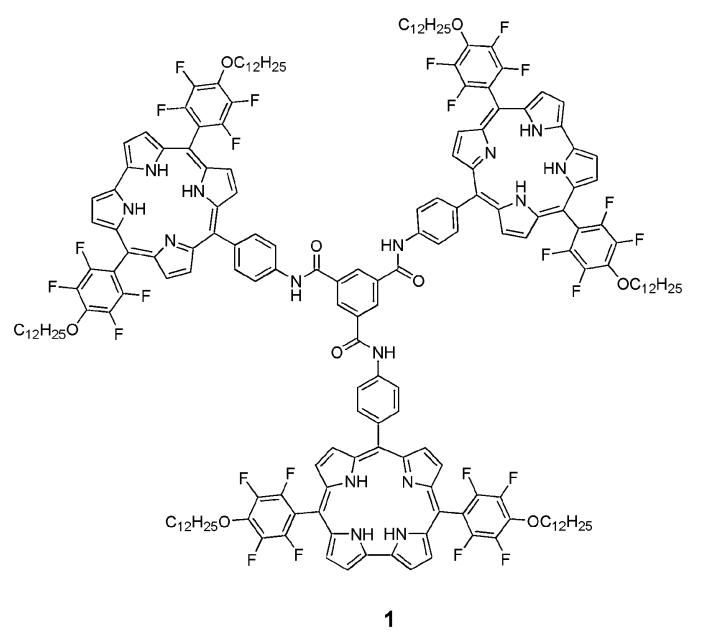

Chart 1 

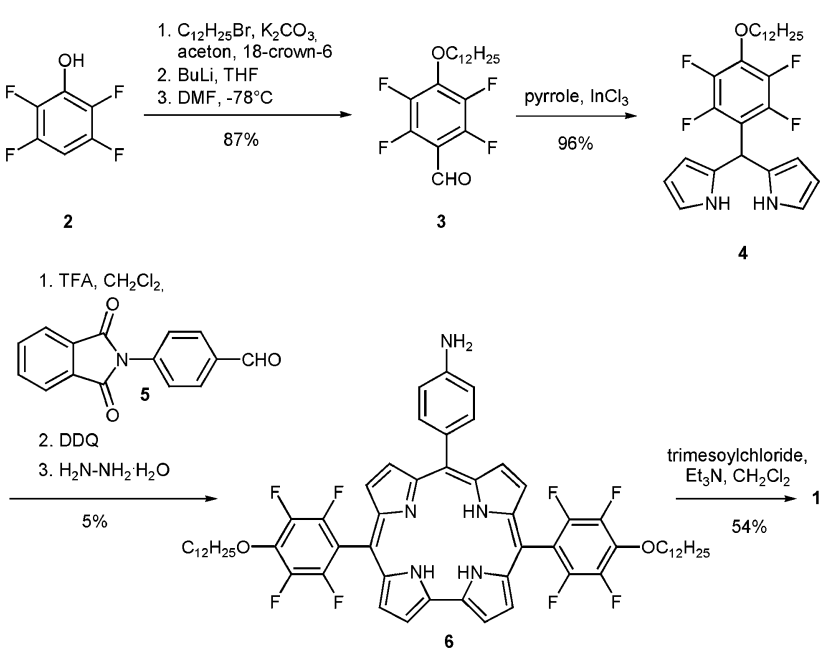

Scheme 1 Synthesis of corrole trimer 1.

size-exclusion column chromatography the compound was obtained in pure form in a yield of 54\%. MALDI-TOF analysis showed a peak at $m / z=3318.4 \mathrm{amu}\left(\mathbf{M}^{+}\right)$, confirming the identity of $\mathbf{1}$.

The corrole rings in $\mathbf{1}$ are coupled to a central trimesic amide core, a motif which is well known for its ability to form extended hydrogenbonded 1D columnar stacks. ${ }^{20-22}$ Although corroles are slightly less planar than porphyrins, ${ }^{23,24}$ it can be expected that, just like in the case of the related porphyrin trimers, ${ }^{10}$ the columnar stacks formed by 1 are further stabilised by $\pi-\pi$ stacking interactions between the corrole side groups (Fig. 1). ${ }^{25}$

The ${ }^{1} \mathrm{H}-\mathrm{NMR}$ spectra of $\mathbf{1}$ in $\mathrm{CDCl}_{3}$ showed only extremely broadened signals, which indicates that at NMR concentrations $\left(>10^{-4} \mathrm{M}\right)$ this compound also self-assembles into columnar stacks. Upon the addition of DMSO- $\mathrm{d}_{6}$ to the sample, the signals sharpened, which is the result of the breaking up the hydrogen bonding network and the dissolution of the stacks. UV-vis spectra of $\mathbf{1}$ in chloroform showed features which are typical for corrole monomers (Fig. 2). ${ }^{16} \mathrm{~A}$ plot of the absorbance maxima of various bands versus the concentration gave straight lines, which indicates that in this solvent in the concentration range of $1-25 \mu \mathrm{M}$ compound $\mathbf{1}$ is molecularly dissolved (Fig. 2B). This means that the critical aggregation constant (c.a.c.) of $\mathbf{1}$ in chloroform lies in between the concentrations used in the UV-vis and NMR experiments. UV-vis spectra of $\mathbf{1}$ in $n$-hexane exhibit a broadened Soret band, with a width at half height of $66 \mathrm{~nm}$, whereas this value in chloroform is only $36 \mathrm{~nm}$ (Fig. 2). The broadening indicates that in $n$-hexane the corrole trimer exists in an aggregated form. The possibility to measure the absorbance of $\mathbf{1}$ as a function of concentration was limited by its precipitation at concentrations above approximately $3 \mu \mathrm{M}$. For this reason, a sample

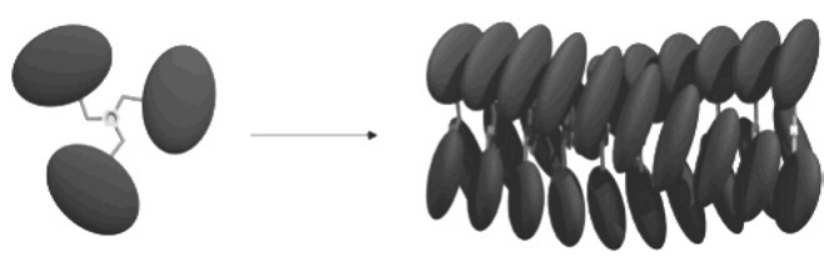

Fig. 1 Schematic representation of the structure of corrole trimer 1 and its self-assembly into a columnar stack.
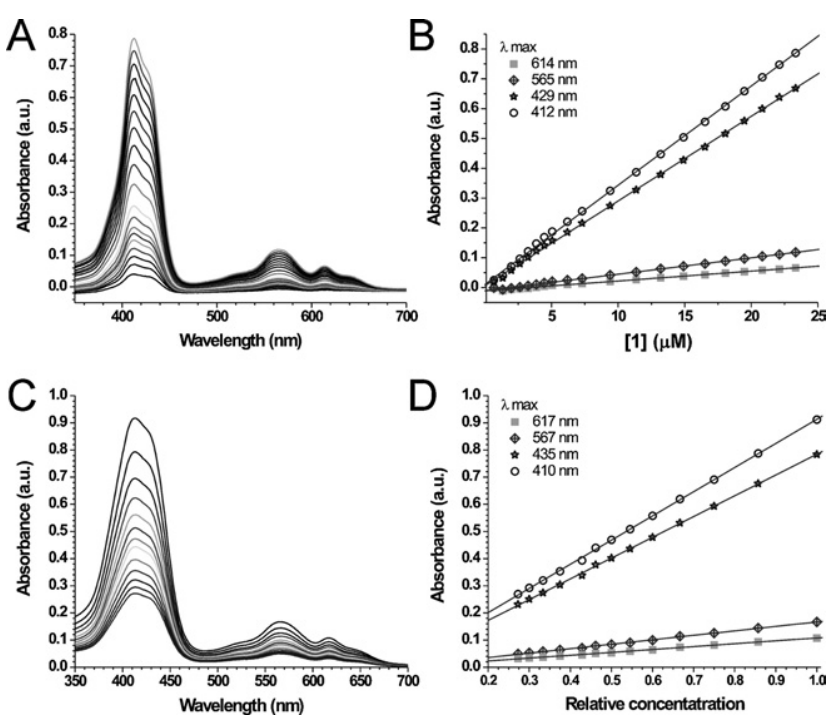

Fig. 2 UV-vis spectra at various concentrations (A, C) and plots of absorbance maxima versus concentration (B, D) of $\mathbf{1}$ in chloroform (A, B) and $n$-hexane (C, D).

of a saturated solution of $\mathbf{1}$ in $n$-hexane was diluted stepwise, and the concentrations of the resulting solutions were expressed relative to the concentration at saturation. During the dilution, the shape of bands in the UV-vis spectra remained unchanged and again linear relationships were observed in the plots of the absorption maxima versus concentration (Fig. 2D). The fact that also in this case Beer's Law is obeyed indicates that the assemblies formed by $\mathbf{1}$ in $n$-hexane are stable, even at very low concentrations. It also implies that the c.a.c. of $\mathbf{1}$ in this solvent is below $10^{-7} \mathrm{M}$.

After evaporation of a solution of 1 in chloroform $(3 \mu \mathrm{l},[\mathbf{1}]=$ $4 \mu \mathrm{M}$ ) which was drop-cast onto a mica surface, contact pinning lines ${ }^{10}$ were observed, which result from the evaporation according to the coffee-stain mechanism ${ }^{26}$ and consist of randomly deposited corrole material. In addition, large domains of patches that typically result from dewetting were present (Fig. 3A,B). ${ }^{27}$ The fact that no epitaxy is observed in the patch structures indicates that the mica surface does not strongly interact with the adsorbed molecules. Remarkably, periodically patterned surfaces of equidistant lines, previously observed for the related porphyrin trimers, ${ }^{10}$ were never observed for the corrole trimer. All over the surface the patches in the dewetting pattern had a highly constant height of $2.9 \pm 0.2 \mathrm{~nm}$ (Fig. 3D and Fig. S1†), a value that corresponds well with the diameter of a molecule of $\mathbf{1}$. This observation indicates that the patches consist of multiple single molecule wide columnar stacks of 1, which are oriented 'edge-on' to the surface, and are probably arranged rather randomly within the dewetted film. Only sporadically, single molecule wide columnar stacks of $\mathbf{1}$ were observed, but in general the assemblies packed into wider patches.

The enhanced tendency of $\mathbf{1}$ to self-assemble in $n$-hexane solution was also reflected in its aggregation behaviour at the mica/n-hexane interface. A saturated solution of 1 in $n$-hexane $(3 \mu \mathrm{l},[1] \approx 3 \mu \mathrm{M})$ was drop-cast onto a mica surface and the solvent was allowed to evaporate. AFM images of the resulting surface revealed that $\mathbf{1}$ had self-assembled into extended fibre-like aggregates with lengths up to several micrometres (Fig. 3C). In contrast to the dewetted structures at the mica/chloroform interface, now a broad variation in the height 

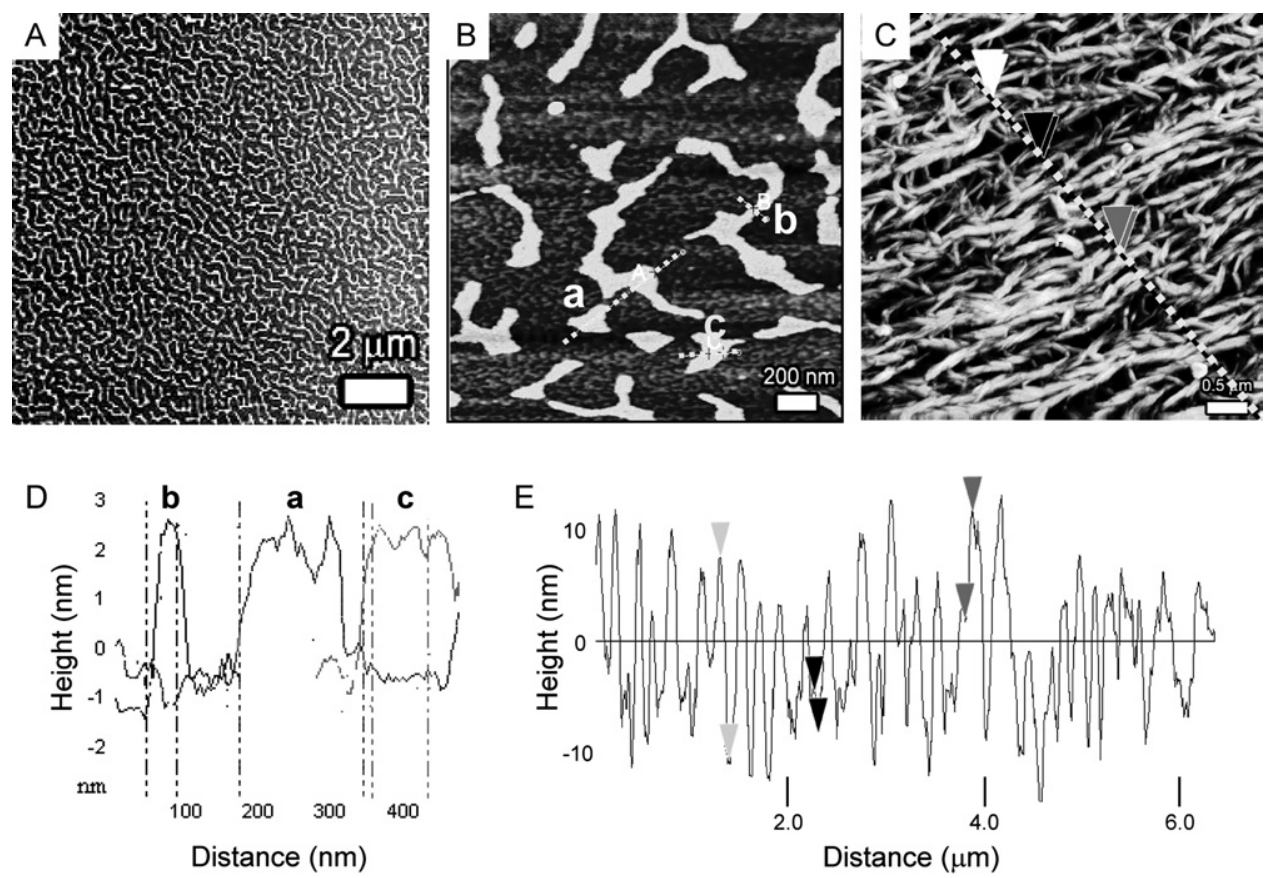

Fig. 3 (A and B) AFM images of patch-like patterns formed upon the evaporation of a chloroform solution of 1 on mica. (C) AFM image of fibre-like aggregates that are deposited from an $n$-hexane solution of $\mathbf{1}$ onto a mica surface. (D) Cross-section of the dashed lines in (B). (E) Cross-section of the dashed line in $(\mathrm{C})$.

of the fibres was observed (Fig. 3E). The smallest height difference observed was $3.5 \mathrm{~nm}$, which corresponds roughly to the diameter of a molecule of $\mathbf{1}$. The observed surface morphology indicates that most of the fibres are built up from thick bundles $(>100 \mathrm{~nm})$ of columnar stacks of $\mathbf{1}$, and the apparent alignment in the AFM image is likely a result of solvent flow arising during evaporation of the $n$-hexane. Despite numerous attempts in which the concentration of the solution was also varied, we did not succeed in isolating fibres composed of a single molecule wide stack of $\mathbf{1}$.

The striking difference in aggregation behaviour of $\mathbf{1}$ at the mica/ chloroform and mica/ $n$-hexane interface is attributed to the difference in self-assembly behaviour in solution. In the droplets that are deposited on the mica, $\mathbf{1}$ is molecularly dissolved in chloroform, while it is aggregated in $n$-hexane. During the dewetting of the thin films the concentration increases in both cases, but selfassembly of $\mathbf{1}$ into columnar stacks in chloroform still has to start while in $n$-hexane already preformed aggregates are present. It is proposed that in chloroform the deposition of $\mathbf{1}$ into single molecule thick patches occurs at the same moment as the columnar stacks are formed, i.e., when the evaporating thin film becomes subject to dewetting. ${ }^{11}$ This mechanism would explain the observation that self-assembly only in two dimensions occurs and not in three, e.g. by the formation of bundles of stacks. The latter is the case for the fibres formed at the mica/n-hexane interface. Since their selfassembly has already occurred in solution, dewetting plays a less important role and the observed surface structures are aggregates of self-assembled columnar stacks that have precipitated from the $n$-hexane film.

Although the aggregation behaviour of $\mathbf{1}$ at the mica/chloroform interface is completely different from that at the mica/n-hexane interface, there is one important similarity between the observed morphologies of the resulting assemblies: in hardly any case were single molecule wide 1D columnar stacks observed. This behaviour is in sharp contrast with the aggregation behaviour of related porphyrin trimers at the same interfaces, where typically single molecule wide stacks and fibers were observed. ${ }^{10,11}$ The concentration of $\mathbf{1}$ in $n$-hexane used for drop-casting is more than a factor of two lower than the concentration used for the porphyrin trimers in $n$-hexane, ${ }^{11}$ which means that the apparent lack of ability of $\mathbf{1}$ to form single molecule thick fibres is not simply due to a concentration effect. The difference in self-assembly between the corrole trimer and the porphyrin trimers most likely finds its origin in the substitution patterns of the chromophoric building blocks. Whereas the porphyrin trimers contain nine meso- $n$-dodecoxyphenyl groups at their periphery, $\mathbf{1}$ has only six. As a result, at the periphery of the columnar stacks of $\mathbf{1}$, the aromatic surfaces of the corrole moieties are available for lateral interactions with adjacent columnar stacks, which can have a stabilising character as a result of $\pi-\pi$ stacking between the columnar stacks. At the mica-chloroform interface, these interactions give only rise to self-assembly in two dimensions, since self-assembly of the molecules takes place only during the dewetting process. In $n$-hexane, aggregation already occurs in solution and it is proposed that, as a result of the lateral interactions, supercoiling occurs in which multiple columnar stacks form thicker fibres (Fig. 4), which precipitate upon evaporation of the solvent.

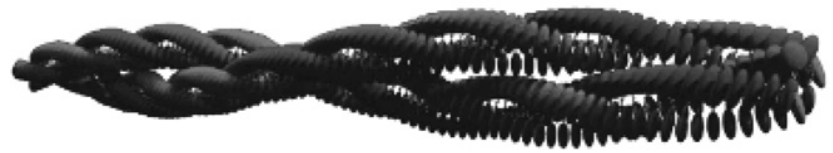

Fig. 4 Schematic representation of the proposed self-assembly of $\mathbf{1}$ into bundles of columnar stacks in $n$-hexane. 
In conclusion, an apparently small change of building block from porphyrin to corrole has a profound effect on the aggregation behaviour of trimesic amide trimers. We proved that self-assembly patterns can be controlled by structural motifs such as amide hydrogen bonding and $\pi-\pi$ interactions between the corrole surfaces, which ensure a strong assembly into 1D columnar stacks, whereas lateral stacking interactions between the corroles in different stacks give rise to further assembly in the second or third dimension. These results are not only of theoretical significance in that they provide new insight into factors influencing self-assembly, but they may also open the door to practical applications. Further research will focus on investigating the (photo)physical properties of the assemblies at the surface.

This work was supported by grants from the Netherlands Organization of Scientific Research (NWO) to J.A.A.W.E. and A.E.R.; the National Research School Combination Catalysis (NRSC-C) to R. van H. and to R.J.M.N.; the Royal Netherlands Academy of Science to R.J.M.N.; and from the Polish Ministry of Science and Higher Eduation to D.T.G.

\section{Notes and references}

1 H. O. Jacobs, A. R. Tao, A. Schwartz, D. H. Gracias and G. M Whitesides, Science, 2002, 296, 323.

2 V. Percec, M. Glodde, T. K. Bera, Y. Miura, I. Shiyanovskaya, K. D. Singer, V. S. K. Balagurusamy, P. A. Heiney, I. Schnell, A. Rapp, H. W. Spiess, S. D. Hudson and H. Duan, Nature, 2002, 419, 384 .

3 V. Palermo and P. Samorì, Angew. Chem. Int. Ed, 2007, 24, 4428.

4 W. R. Browne and B. L. Feringa, Nat. Nanotech, 2006, 1, 25.

5 D. K. James and J. M. Tour: Self-Assembled Molecular Electronics. In: Nanoscale Assembly Techniques; W. T. S. Huck, Ed.; Springer: New York, 2005; pp 79-98.

6 H. Tokuhisa and P. Hammond, Adv. Funct. Mater, 2003, 13, 831.

7 B. Hulsken, R. van Hameren, J. W. Gerritsen, T. Khoury, P. Thordarson, M. J. Crossley, A. E. Rowan, R. J. M. Nolte, J. A. A. W. Elemans and S. Speller, Nat. Nanotech, 2007, 2, 285.

8 L. Piot, D. Bonifaci and P. Samorì, Adv. Funct. Mat., 2007, 17, 3689.

9 J. A. A. W. Elemans, R. van Hameren, R. J. M. Nolte and A. E. Rowan, Adv. Mater, 2006, 18, 1251.

10 R. van Hameren, P. Schön, A. M. van Buul, J. Hoogboom, S. V. Lazarenko, J. W. Gerritsen, H. Engelkamp, P. C. M. Christianen, H. A. Heus, J. C. Maan, Th. Rasing, S. Speller, A. E. Rowan, J. A. A. W. Elemans and R. J. M. Nolte, Science, 2006, 314, 1433.
11 R. van Hameren, A. M. van Buul, M. A. Castriciano, V. Villari, N. Micali, P. Schön, S. Speller, L. Monsù Scolaro, A. E. Rowan, J. A. A. W. Elemans and R. J. M. Nolte, Nano Lett., 2008, 8, 253.

12 (a) B. Ventura, A. Degli Esposti, B. Koszarna, D. T. Gryko and L. Flamigni, New J. Chem., 2005, 29, 1559; (b) A. Rebane, M. Drobizhev, N. S. Makarov, B. Koszarna, M. Tasior and D. T. Gryko, Chem. Phys. Lett, 2008, 462, 365.

13 T. Ding, E. A. Aleman, D. A. Modarelli and C. J. Ziegler, J. Phys. Chem. A, 2005, 109, 7411.

14 J. Shen, J. Shao, Z. Ou, W. E. B. Koszarna, D. T. Gryko and K. M. Kadish, Inorg. Chem., 2006, 45, 2251.

15 L. Flamigni, B. Ventura, M. Tasior, T. Becherer, H. Langhals and D. T. Gryko, Chem. Eur. J, 2008, 14, 169.

16 B. Koszarna and D. T. Gryko, J. Org. Chem, 2006, 71, 3707.

17 D. T. Gryko and B. Koszarna, Synthesis, 2004, 2205.

18 J. K. Laha, S. Dhanalekshmi, M. Taniguchi, A. Ambroise and J. S. Lindsey, Org. Proc. Res. Dev., 2003, 7, 799.

19 M. Tasior, D. T. Gryko, M. Cembor, J. S. Jaworski, B. Ventura and L. Flamigni, New J. Chem., 2007, 31, 247.

20 M. P. Lightfoot, F. S. Mair, R. G. Pritchard and J. E. Warren, Chem. Commun., 1999, 1945.

21 M. L. Bushey, T. Q. Nguyen, W. Zhang, D. Horoszewski and C. Nuckolls, Angew. Chem. Int. Ed., 2004, 43, 5446.

22 A. J. Wilson, M. Musada, R. P. Sijbesma and E. W. Meijer, Angew. Chem. Int. Ed., 2005, 44, 2275.

23 T. Ding, J. D. Harvey and C. J. Ziegler, J. Porphyrins Phthalocyanines, 2005, 9, 22.

24 A. M. Albrett, J. Conradie, A. Ghosh and P. J. Brothers, Dalton Trans., 2008, 4464.

25 Other examples of supramolecular columnar stacks in which $\pi-\pi$ interactions play a stabilising role: (a) T.-Q. Nguyen, R. Martel, M. Bushey, P. Avouris, A. Carlsen, C. Nuckolls and L. Brus, Phys Chem. Chem. Phys, 2007, 9, 1515; (b) R. I. Gearba, A. Bondar, M. Lehmann, B. Goderis, W. Bras, M. H. J. Koch and D. A. Ivanov, Adv. Mater, 2005, 17, 671; (c) M. Lehmann, R. I. Gearba, M. H. J. Koch and D. A. Ivanov, Chem. Mater., 2004, 16, 374; (d) H. M. Keizer and R. P. Sijbesma, Chem. Soc. Rev., 2005, 34, 226; (e) V. Percec, M. Glodde, T. K. Bera, Y. Miura, I. Shiyanovskaya, K. D. Singer, V. S. K. Balagurusamy, P. A. Heiney, I. Schnell, A. Rapp, H. W. Spiess, S. D. Hudson and H. Duan, Nature, 2002, 419, 384; (f) R. S. Johnson, T. Yamazaki, A. Kovalenko and H. Fenniri, J. Am. Chem. Soc., 2007, 129, 5735 .

26 R. D. Deegan, Phys. Rev. E, 2000, 61, 475.

27 For some examples of (dewetted) patterns of polymeric materials, see: (a) C. Park, J. Yoon and E. L. Thomas, Polymer, 2003, 44, 6725; (b) S. B. Darling, Prog. Polym. Sci., 2007, 32, 1152; (c) H. Yabu and M. Shimomura, Adv. Funct. Mat., 2005, 15, 575; (d) B. H. Kim, D. O. Shin, S.-J. Jeong, C. M. Koo, S. C. Jeon, W. J. Hwang, S. Lee, M. G. Lee and S. O. Kim, Adv. Mater., 2008, 20, 2303. 